\title{
Agôn
}

Revue des arts de la scène

Critiques | Saison 2013-2014

\section{Die gelbe Tapete de Katie Mitchell et Les Marchands de Joël Pommerat}

comme au cinéma?

\section{Marion Boudier}

\section{(2) OpenEdition}

Journals

Édition électronique

URL : http://journals.openedition.org/agon/2720

DOI : 10.4000 /agon. 2720

ISSN : 1961-8581

Éditeur

Association Agôn

Référence électronique

Marion Boudier, « Die gelbe Tapete de Katie Mitchell et Les Marchands de Joël Pommerat », Agôn [En ligne], Critiques, mis en ligne le 10 octobre 2013, consulté le 23 septembre 2020. URL : http:// journals.openedition.org/agon/2720; DOI : https://doi.org/10.4000/agon.2720

Ce document a été généré automatiquement le 23 septembre 2020.

Association Agôn et les auteurs des articles 


\title{
Die gelbe Tapete de Katie Mitchell et Les Marchands de Joël Pommerat
}

\author{
Comme au cinéma?
}

Marion Boudier

\section{RÉFÉRENCE}

Die gelbe Tapete de Katie Mitchell et Les Marchands de Joël Pommerat. A l'Odéon-Théâtre de l'Europe, septembre 2013.

1 Accueillis au même moment dans les deux salles de l'Odéon-Théâtre de l'Europe à Paris, Die gelbe Tapete de Katie Mitchell et Les Marchands de Joël Pommerat invitent à aborder à nouveau la question, devenue récurrente dans l'esthétique théâtrale contemporaine, des relations entre théâtre et cinéma, et plus largement celle des liens entre récit et image, leurs raccords concrets ou imaginaires sur scène et dans la tête des spectateurs.

2 Au delà de similitudes anecdotiques (les protagonistes principaux sont des femmes, il y a deux enfants plus ou moins sacrifiés, il y a des apparitions, des murs trop nus ou trop couverts de papier peint), ces deux spectacles traitent d'une question de perception et de représentation: conscience de soi altérée par le chavirement intime du baby blues dans Die gelbe Tapete inspiré d'une nouvelle de Charlotte Perkins Gilman; conscience altérée par l'intériorisation excessive d'un certain discours sur la valeur du travail dans Les Marchands. En jouant sur la dissociation des différents éléments de la représentation (texte, son, image), ces deux spectacles proposent aux spectateurs deux expériences perceptives et réceptives originales, leurs dispositifs scéniques redoublant en quelque sorte leurs contenus et thématiques.

3 Ce que nous voudrions préciser dans cette critique en forme de comparaison et d'études de cas des deux œuvres, c'est comment Katie Mitchell reproduit le cinéma en scène alors que Joël Pommerat produit sur le spectateur un «effet cinéma». L'une travaille avec l'image cinématographique, l'autre stimule à partir de l'image scénique le film intérieur du spectateur, ses images mentales. 
Die gelbe Tapete de Katie Mitchell

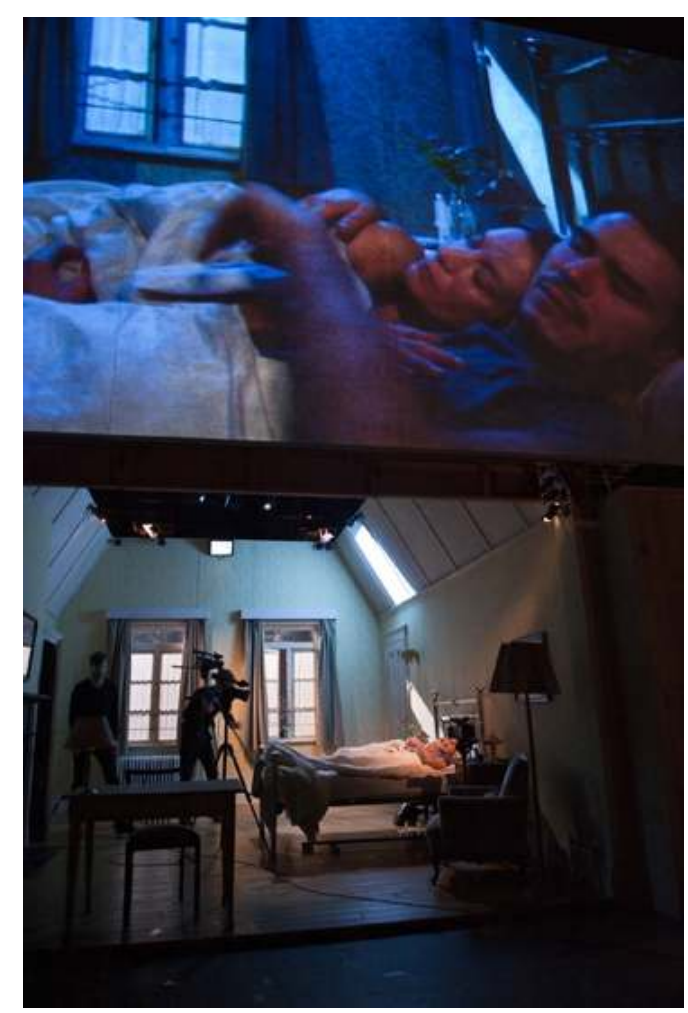

(C) Stephen Cummiskey

Katie Mitchell introduit le cinéma au théâtre en filmant la scène en direct. Comme elle l'a fait avec Christine (d'après Mlle Julie de Strindberg) puis Reise durch die nacht (d'après Frederike Mayrücker) présenté au Festival d'Avignon en 2011 et 2013, dans Die gelbe Tapete, les acteurs sont filmés pendant qu'ils jouent. Le film de leurs actions est projeté sur un grand écran suspendu au-dessus de l'espace de jeu, qui est donc aussi un espace de tournage où évoluent les cameramen, où sont déplacés les meubles - sous nos yeux mais hors du cadre de la caméra. Côté jardin se trouve une cabine de bruitage et de montage. Au spectateur de choisir que regarder: le film ou le film en train d'être fabriqué, l'image ou l'élaboration de cette représentation. Ce faisant il lui est offert la possibilité de voir non seulement le hors champ mais aussi le hors cadre. En revanche, les coulisses théâtrales de ce plateau de tournage sont peu visibles. À certains moments, de grands panneaux de bois masquent complètement l'espace de tournage au profit de la seule image filmée. Moments qui laissent sentir que la finalité de ce théâtre, c'est le film et ses gros plans plus que la fabrication du film.

Pourquoi alors proposer au spectateur ce double regard sur la représentation cinématographique et sa fabrication? Il n'y a pas de jeu de décalages entre le plateau et son image, si ce n'est la différence entre notre vision frontale de la scène et les différents angles de vue des caméras ainsi que certains plans tournés hors scène (ou plutôt sur la scène hors la vue). L'écoute, la vision des comédiens en train de jouer et la vision du film sont trois opérations de perception proposées conjointement et en harmonie, sans contrepoint ni dissensus. La distance vient d'un autre film, un film « d'archive » tourné par le père, Christophe, qui présente quelques moments de leur vie d'avant la dépression. Ce film amateur se substitue à plusieurs reprises au film du 
direct du plateau selon une dynamique de flashback, mais monté à rebours: il commence par une scène qui montre Anna (Judith Engel) avec une poussette loin derrière son fils que porte une tante, exubérante d'amour pour l'enfant, et il s'achève avec l'arrivée à la maison au sortir de la maternité, lorsqu'Anna passe la porte, souriante, en portant le couffin. À la dégradation progressive de l'état mental de la jeune femme présentée en direct répond cette remontée vers un temps des origines heureuses, marche inversée qui provoque un effet de contraste saisissant.

Le dispositif du tournage et du bruitage en direct suscite curiosité et admiration pour sa prouesse, mais il occupe globalement beaucoup de place et, à moins d'être assis dans les dix premiers rangs des gradins, l'aura de l'écran géant l'emporte pour le spectateur sur l'observation de la scène. Pourtant ce dispositif « méta » ne lasse pas, en raison du redoublement de l'effet performatif qu'il produit, effet du direct et effet de présence. Cela est particulièrement sensible à la fin du spectacle, écrit en crescendo: lorsque Christophe (Tilman Strauss) défonce la porte de la chambre et se précipite au secours de sa femme, sentir le mouvement de sa course sur le plateau, le bruit de ses pas, son souffle, la vibration des cloisons, donne une intensité remarquable à l'image cinématographique. Une image augmentée de présence.

Le suspens de la situation (Anna guidée jusqu'à sa baignoire par une mystérieuse jeune femme prisonnière de la tapisserie qu'elle a lacérée toute la nuit enfin de la libérer, sèche cheveux en main, porte verrouillée à clef) est subrepticement redoublé par cette étonnante pensée fugace : Christophe va-t-il courir aussi vite que son image ? Question absurde puisque le personnage est filmé en direct, mais qui souligne le jeu étonnant des différents éléments entre eux !

Die gelbe Tapete de Katie Mitchell

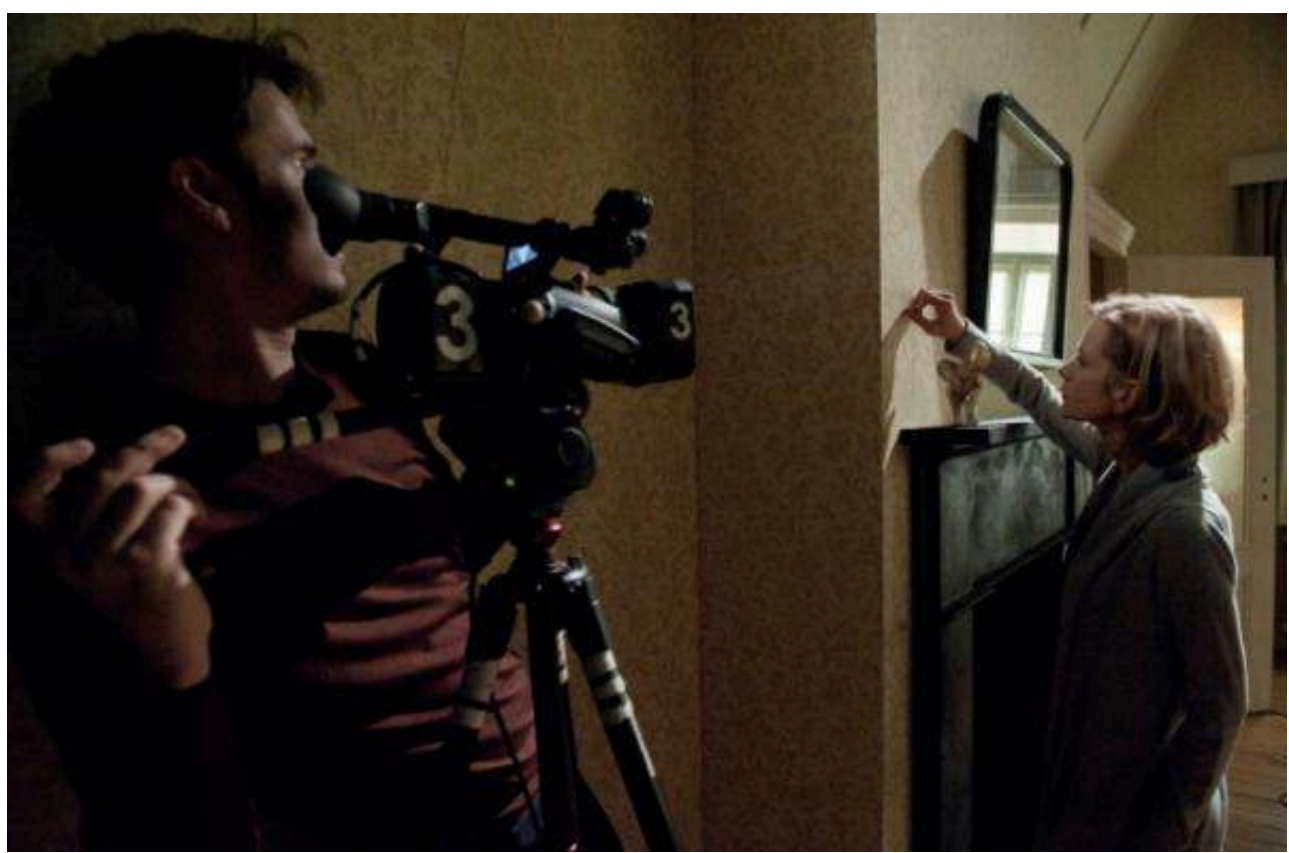

(C) Stephen Cummiskey

La dissociation de l'image d'Anna et du son de sa voix, (monologue intérieur remarquablement interprété dans une cabine vitrée située au centre du plateau par Ursina Lardi) participe du même effet d'intensification des différents langages présents 
sur la scène. La disjonction parfaitement synchronisée des différentes composantes de la représentation produit donc au niveau de la captation du spectateur un effet plus fort que celui de leur fusion, en redonnant à chacune une intensité particulière. Cette intensité dépend in fine de la sensibilité de chaque spectateur, de sa propre activité de perception, de son travail d'assemblage et de montage des différents éléments (corps, son, image) entre eux.

Dans ce spectacle, le théâtre ne modifie pas le cinéma, il est au service du film. Pas de noir, pas de long plan fixe frontal, pas de changement de décor dans le film comme on peut en voir au théâtre. Le film en revanche, modifie la théâtralité. Certes il exalte la qualité du jeu en donnant à voir les excellents acteurs de la Schaubühne en gros plans, mais c'est parce que son tournage implique aussi un jeu clos sur lui-même, derrière un quatrième mur étanche, barrière entre la scène et la salle que renforce la présence des cameramen vêtus de noir. L'adresse au public est maintenue, in extremis, par le regard de la diseuse ("pensées d'Anna ») qui nous fait face. Mais dans l'ensemble, le réalisme filmique emporte l'attention du spectateur, conformément à ses habitudes audiovisuelles (visibilité, montage rapide). La réalisation du film polarise donc la représentation théâtrale, même si le réel du plateau continue de jouer par le surcroît d'intensité que la présence des acteurs donne à l'image.

Les Marchands de Joël Pommerat

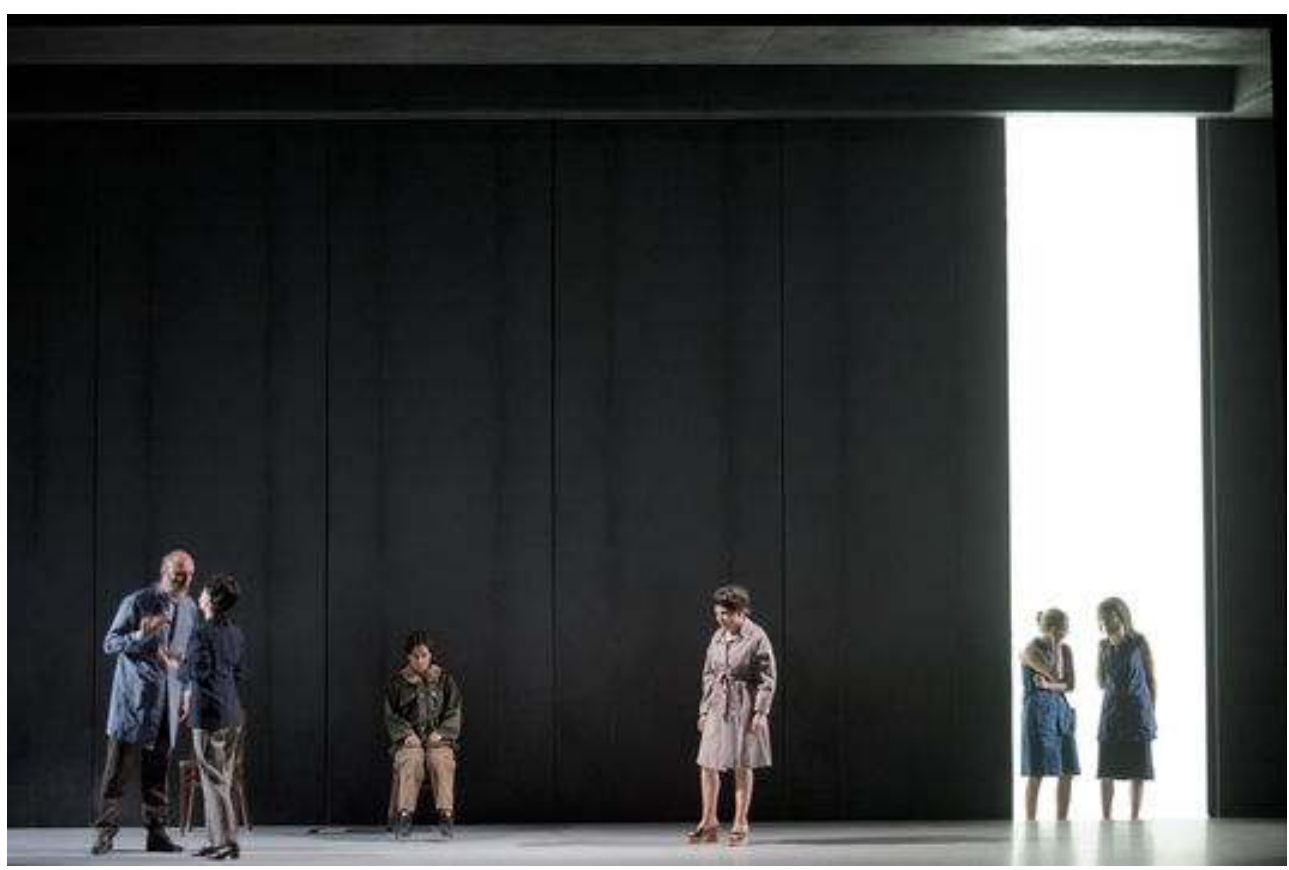

(c) Elisabeth Carecchio

Dans Les Marchands, il n'est pas concrètement fait usage de la technique cinématographique : aucun écran, ni caméra ni image projetée. Le cinéma n'entre pas en jeu comme un dispositif technique et scénique visible mais plutôt comme une référence et un effet de réception. D'où vient cette impression ressentie face aux Marchands par certains spectateurs d'être au théâtre comme au cinéma ${ }^{1}$ ?

Le spectacle est construit selon une dissociation entre, d'une part, le récit d'une ouvrière sur son amour du travail, le devenir de son usine et de sa meilleure amie au 
chômage, et d'autre part, une succession d'images scéniques qui illustrent plus ou moins ce récit. Celui-ci nous parvient la plupart du temps en voix off, mais la narratrice est également présente à certains moments, intégrée à la situation de jeu même si elle est placée un peu plus à l'avant-scène que les autres personnages. Dans ce spectacle, la dissociation entre la voix et les images prend une dimension critique. Le récit monocorde, répétitif, lacunaire, sans contradiction ni point de vue adverse, est certes entraînant et suscite l'adhésion, mais dans la durée il se métamorphose en ressassement absurde. Et cela d'autant plus que l'apologie du travail faite par la narratrice est mise en accusation par la vue de son corps enserré dans un corset et une minerve. Témoin de cette tension entre un état, sa perception et sa justification, le spectateur oscille entre la compassion, la compréhension et l'indignation ou la conscience critique. La justesse avec laquelle la souffrance humaine est montrée et la précision des images captent l'attention. Cette captation est aussi d'ordre sensible, liée aux sensations produites par la lumière et le son. Sans discuter ici plus avant les enjeux critiques de l'esthétisation du travail et de la misère à l'œuvre dans le spectacle, c'est le pouvoir cinématographique de ces images sensibles que nous souhaitons interroger.

\section{Les Marchands de Joël Pommerat}

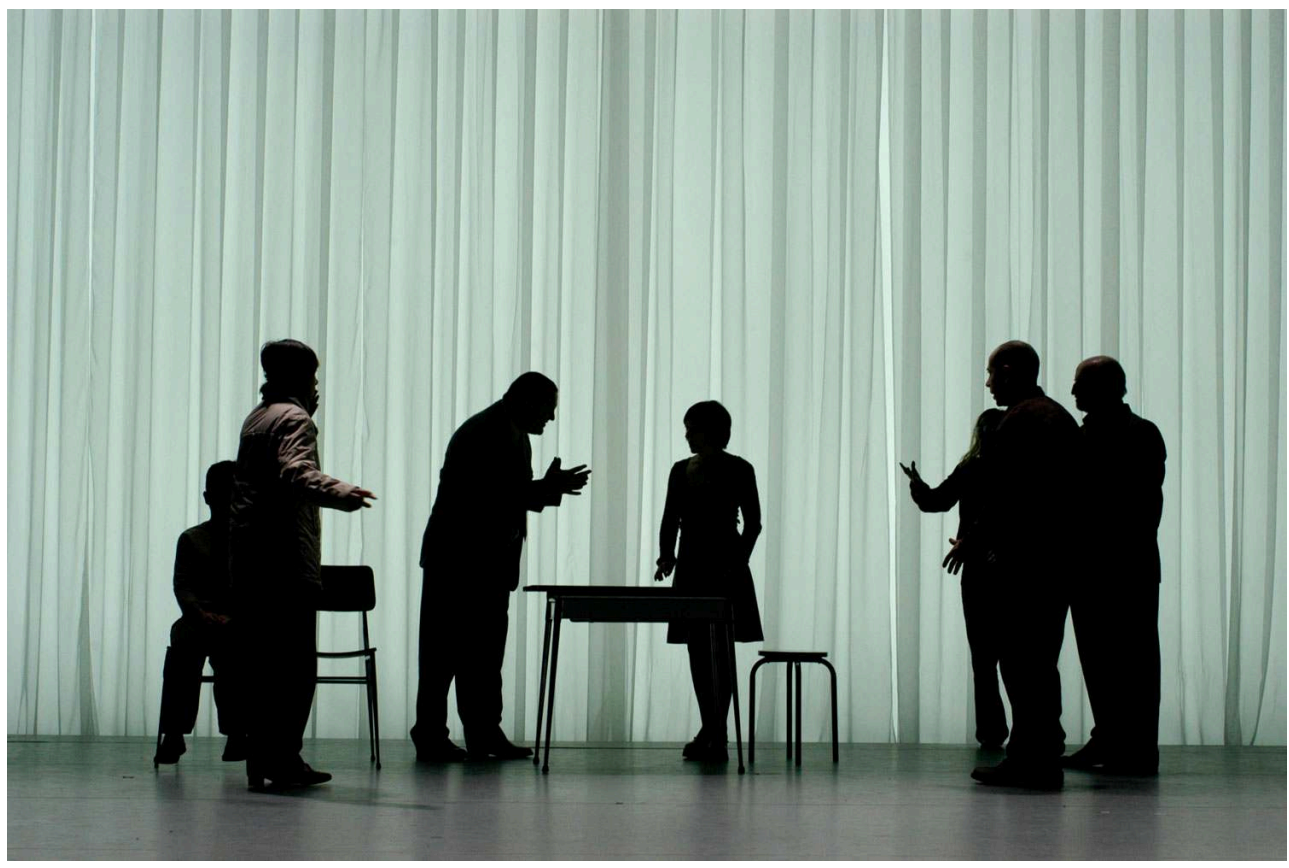

(c) Elisabeth Carecchio

D'un point de vue dramaturgique, Joël Pommerat recourt à certains éléments que l'on associe à la grammaire cinématographique (même si ces éléments appartiennent tout autant au vocabulaire du théâtre ou à celui du roman), comme le montage, les ellipses et une grande liberté narrative. L'agencement des scènes des Marchands qui oscillent entre réalité sociale et apparitions spirites peut évoquer certains jeux formels des films de David Lynch. Mais la «sensation de cinéma" ressentie face au spectacle tient surtout à sa mise en scène. Les spectateurs sont plongés dans un noir profond (moins sensible cependant depuis balcons et corbeilles à l'Odéon), qui évoque celui d'une salle de cinéma. Dans la lumière froide à dominante blanche, grise et bleue qui enveloppe la scène, le jeu muet des acteurs et leur gestuelle précise, à la fois stylisée et chargée de 
présence, éloquente, rappellent les films en noir et blanc de Dreyer. Les images s'enchaînent comme des plans séquences ponctuées de courts noirs. La rapidité de cet enchaînement, avec ses entrées ou fondus au noir sec qui évoquent des cuts, possède une grande fluidité qui tend à créer l'impression du mouvement propre à la succession des séquences d'un film.

En travaillant sur de basses intensités lumineuses, la pénombre et des contrastes ou contre-jours, le scénographe et éclairagiste Eric Soyer stimule notre perception visuelle et réussit à faire sentir comme le grain de l'image filmée. Cet effet est notamment renforcé par la dimension de la baie vitrée de l'appartement de l'amie qui évoque le format cinémascope et dont la luminosité nous éclaire comme un écran placé face à nous. Le travail de la lumière crée également du mouvement, en donnant l'impression d'un rapprochement vers un personnage ou celle d'un gros plan sur lui grâce à une découpe lumineuse. Au début du spectacle, lorsque l'amie, dont la silhouette noire se détache en fond de scène sur une lumière blanche, tire sur toute la longueur du plateau, de jardin à cour, un panneau noir qui fait passer à l'image suivante, le spectateur peut avoir l'illusion d'un effet de travelling. L'absorption du spectateur dans l'image est soutenue par son immersion sonore. La bande son est omniprésente, voix off, bruitages, musique, chansons. Pendant les noirs qui séparent les séquences, le son prolonge l'image et stimule l'imagination des spectateurs.

Sensation physique liée à la lumière et au son, à un travail sur le cadre et sur la composition de l'image, le cinéma des Marchands est donc dans la tête de ses spectateurs. Entre réalisme et abstraction, l'épuration des images scéniques au sein même de leur grande précision fonctionne comme une suggestion, un espace à la fois assez déterminé et ouvert pour devenir une surface de projection mentale : comme lorsqu'il lit un texte et élabore des images mentales des personnages qui y sont décrits et mis en intrigue, le spectateur réalise ainsi lui-même le film du spectacle en complétant l'image de ses propres références et représentations. "Son appartement, / là où elle habitait, / c'était, / vous ne pouvez pas vous imaginer, / le plus grand vide matériel qu'on eût pu redouter pour quelqu'un,/ car c'était le vide... ${ }^{2}$ ». Le vide stimule l'imagination de chacun et c'est en chaque spectateur que se cristallise une impression de réalité aussi forte, bien que distincte, que celle produite par l'image cinématographique. Cet effet de réel nous semble comparable à celui du cinéma en raison de la parfaite maîtrise de l'image scénique orchestrée par Joël Pommerat et ses acteurs: la prise de risque théâtrale disparaît, la fragilité et l'inégalité du travail de l'acteur sont oubliées, on est en face de la bonne prise, la seule qui sera conservée sur la pellicule. Même si elle peut être étrange et dérangeante, cette image est sûre et rassurante comme celle du cinéma ; l'erreur, la panne ou le trou semblent impossibles. Et pourtant la jubilation du réel demeure, sans le stress, à travers l'intense présence des comédiens qui crèvent l'écran de nos scènes imaginaires. 


\section{NOTES}

1. Voir aussi Marion Boudier, " "Je ne pense pas cinéma quand je fais du théâtre" : influences et effets cinématographiques chez Joël Pommerat », in Marguerite Chabrol, Tiphaine Karsenti, Théâtre et cinéma : le croisement des imaginaires, PUR, col. Le Spectaculaire, 2013, pp. 219-232.

2. Joël Pommerat, Les Marchands, Arles, Actes Sud - Papiers, 2006, p. 8. 